Portland State University

PDXScholar

Mathematics and Statistics Faculty

Fariborz Maseeh Department of Mathematics

Publications and Presentations

and Statistics

1999

\title{
Geometrical Models for Grain Dynamics
}

Giovani L. Vasconcelos

Universidade Federal de Pernambuco

J. J. P. Veerman

Portland State University, veerman@pdx.edu

Follow this and additional works at: https://pdxscholar.library.pdx.edu/mth_fac

Part of the Dynamical Systems Commons, and the Geometry and Topology Commons Let us know how access to this document benefits you.

\section{Citation Details}

Vasconcelos, Giovani L. and Veerman, J. J. P., "Geometrical Models for Grain Dynamics" (1999).

Mathematics and Statistics Faculty Publications and Presentations. 148.

https://pdxscholar.library.pdx.edu/mth_fac/148

This Post-Print is brought to you for free and open access. It has been accepted for inclusion in Mathematics and Statistics Faculty Publications and Presentations by an authorized administrator of PDXScholar. Please contact us if we can make this document more accessible: pdxscholar@pdx.edu. 


\title{
Geometrical Models for Grain Dynamics
}

\author{
Giovani L. Vasconcelos ${ }^{1}$ and J. J. P. Veerman ${ }^{2}$ \\ ${ }^{1}$ Departamento de Física \\ Universidade Federal de Pernambuco \\ 50670-901, Recife, Brazil \\ ${ }^{2}$ Mathematics Department \\ Georgia Institute of Technology, Atlanta, USA
}

April 9, 1999

\begin{abstract}
We study models for the gravity-driven, dissipative motion of a single grain on an inclined rough surface. Imposing some conditions on the momentum loss due to the collisions between the particle and the surface, we arrive at a class of models in which the grain dynamics is described by one-dimensional maps. The dynamics of these maps is studied in detail. We prove the existence of various dynamical phases and show that the presence of these phases is independent of the restitution law (within the class considered).
\end{abstract}

\section{INTRODUCTION}

Recently, we proposed [1] a model for the dynamics of a single grain on a rough inclined surface. The model had some remarkable properties which enabled us to carry out a detailed description of its dynamics. The results we obtained were consistent with the limited evidence from experiments [2]. In this paper we will show that the qualitative aspects of the dynamics are insensitive to the actual form of the tangential restitution law. Thus, we generalize our previous results reported in Ref. [1], which were derived assuming one particularly simple form of the restitution law.

In our model, which is shown in Fig. 1, the rough surface is considered to have a simple staircase shape whose steps have height $a$ and length $b$. For convenience, we choose a system of coordinates in such a way that the step 
plateaus are aligned with the $x$ axis and the direction of the acceleration of gravity $\mathrm{g}$ makes an angle $\phi$ with the $y$ axis. A point particle is then imagined to be launched on the top of the 'staircase' with a given initial velocity. Upon reaching the end of a step plateau, the particle will undergo a ballistic flight until it collides with another plateau located a certain number $n$ of steps below the departure step (e.g., $n=3$ in Fig. 1). Accordingly, we will refer to the integer $n$ as the jump number associated with this flight. After the collision, the particle will slide frictionlessly along the step with which it collided until reaching its end, when a new flight begins.

We thus have 3 restrictive assumptions on the model:

1. The form of the tangential restitution law.

2. The absence of normal bouncing.

3. The rectangular shape of the staircase.

The aim of this paper is to lift the first of these restrictions. We will show that our results are robust with respect to the tangential restitution law. This is of importance since tangential restitution laws are very hard to establish experimentally [?] and are not well understood theoretically. As discussed below, we will assume here that the velocity after a collision is an unknown function (of the incoming velocity) satisfying some reasonable requirements. We will then show that all qualitative results obtained in Ref. [1] still hold for this general class of restitution laws.

As far as the other restrictions are concerned, computer simulations [4] have indicated that a nonzero normal coefficient of restitution does not significantly alter the general dynamics of the particle. We will take up this issue in a future work [5]. The third restriction, on the other hand, turns out to be relevant. One can show, however, that the qualitative aspects of the dynamics are unchanged so long as the faces of the steps on which the particle falls all have exactly the same constant slope. We shall see below that the 'staircase' geometry leads to a crucial simplification of the dynamics. The extent to which this influences the dynamics will be discussed in future work.

Now we specify the collision rules. Let $\mathbf{v}=\left(v_{x}, v_{y}\right)$ denote the components of the particle velocity parallel and perpendicular to the collision plane before a collision, respectively, then we will take the velocity $\mathbf{v}^{\prime}=\left(v_{x}^{\prime}, v_{y}^{\prime}\right)$ after the collision to be given by

$$
v_{x}^{\prime}=e_{t} C\left(v_{x}, v_{y}\right)
$$




$$
v_{y}^{\prime}=-e_{n} v_{y}
$$

where $e_{t} C\left(v_{x}, v_{y}\right)$, with $0 \leq e_{t}<1$, represents our generic tangential restitution law and $0 \leq e_{n}<1$ is the normal coefficient of restitution. In Ref. [1], the function $C\left(v_{x}, v_{y}\right)$ was taken to be equal to $v_{x}$ and $e_{n}$ was set to 0 . Here $C$ will only be required to satisfy some reasonable criteria. But for simplicity $e_{n}$ will still be kept zero, the advantage being that the model can then be described by a one-dimensional map. When $e_{n}>0$ the dynamics is governed by a three-dimensional map, the analysis of which is more complicated and will be left for forthcoming publications [5]. We will also neglect the energy dissipation as the particle slides along a step, since we suppose that the main energy loss is due to collisions.

\section{THE MODEL}

We now turn to the exact formulation of our model. For physical reasons, we will require the function $C(x, y)$ to satisfy the following conditions:

1. $C(x, y)$ is twice differentiable and homogeneous of degree 1 , that is, $C(\beta x, \beta y)=\beta C(x, y)$ for $\beta>0$.

2. $C(0,1)=0$.

3. $\partial_{u} C(u, 1) \in[0,1]$.

The physical meaning of the conditions above should be evident. The homogeneity condition implies that, for a fixed angle of incidence, the kinetic energy of the particle afterwards is proportional to its value before the impact. The second condition says that a particle colliding vertically does not acquire tangential momentum, while the third condition ensures a net loss of tangential momentum upon collision, i.e., $v_{x}^{\prime}<v_{x}$.

Let us write $E=\frac{1}{2} m V^{2}$, where $m$ is the particle mass and $V$ is the launching velocity at the start of a flight, and let $E^{\prime}$ denote the corresponding kinetic energy at the beginning of the next flight (see Fig. 1). Using simple arguments of energy conservation together with the collision conditions (1) and (2) [with $e_{n}=0$ ], one can write $E^{\prime}$ in terms of $E$. The result is

$$
E^{\prime}=\frac{1}{2} m\left[e_{t} C\left(v_{x}, v_{y}\right)\right]^{2}+m g \sin \phi(n b-x),
$$


where $n$ is the corresponding jump number for the flight and $x$ is the $x$ coordinate of the landing point. Introducing the notation:

$$
\begin{aligned}
& \mathcal{E}=\frac{E}{m g a \cos \phi}, \\
& g(u)=[C(u, 1)]^{2}, \\
& t=\tan \phi, \\
& \tau=\tan \alpha,
\end{aligned}
$$

we obtain that the dynamics of the model, in terms of the dimensionless variable $\mathcal{E}$, is given by the following map:

$$
\mathcal{E}^{\prime}=f(\mathcal{E}, n)=n e_{t}^{2} g(\sqrt{\mathcal{E} / n}+t)+n t(\tau-t-2 \sqrt{\mathcal{E} / n}) .
$$

The jump number $n$ is determined by the energy $\mathcal{E}$ according to the following condition: $n$ is equal to the smallest integer such that

$$
n(\tau-t)-2 \sqrt{n \mathcal{E}} \geq 0 .
$$

This means that $\mathcal{E}$ falls within the interval $I_{n}$ :

$$
\mathcal{E} \in I_{n}(t) \equiv\left(\frac{1}{4}(n-1)(\tau-t)^{2}, \frac{1}{4} n(\tau-t)^{2}\right] .
$$

Thus the function $f(\mathcal{E}, n)$ exhibits jump discontinuities at energy values $\mathcal{E}=\frac{1}{4} n(\tau-t)^{2}$, but each of its branches is smooth.

From hereon, we will use the notation $z=\sqrt{\mathcal{E} / n}$. In terms of the variable $z$ the map (8) yields an equivalent dynamical system:

$$
z^{\prime}=\sqrt{\frac{n}{n^{\prime}}} h(z)
$$

where owing to the homogeneity of $f(\mathcal{E}, n)$ the function $h(z)$ is independent of $n$, being given by

$$
h(z)=\sqrt{e_{t}^{2} g(z+t)+t(\tau-t-2 z)},
$$

and $z$ takes value in the rescaled interval $J_{n}$

$$
z \in J_{n}(t) \equiv\left(\frac{1}{2} \sqrt{1-n^{-1}}(\tau-t), \frac{1}{2}(\tau-t)\right] .
$$


Note that the homogeneity of $f(\mathcal{E}, n)$, which allowed us to formulate the problem in terms of the variable $z$, is a consequence of the geometry of the staircase and of the homogeneity requirements on $C$. This alternative formulation will turn out to be very useful in the analysis that follows.

\section{FIXED POINTS}

To investigate the existence of fixed points it suffices to study the dynamics within a given interval $J_{n}$, so we set $n^{\prime}=n$. In this case the map (11) becomes simply

$$
z^{\prime}=h(z)
$$

with $z$ restricted to the interval $J_{n}$. The fixed points are then given by the solutions of the equation $z=h(z)$, which in view of (12) gives

$$
(z+t)^{2}=e_{t}^{2} g(z+t)+t \tau .
$$

From the requirements on $C$ it is easy to show that the solution of Eq. (15) is always unique. So call this solution $z_{0}(t)$. Then note that a fixed point with jump number $n$ will exist if $z_{0}(t) \in J_{n}$. We claim that that $z_{0}(t)$ will cross both endpoints of the intervals $J_{n}(t)$ as $t$ increases from 0 to $\tau$. To show this, let us define the quantity $D(t)=z_{0}(t)-z_{\max }(t)$, where $z_{\max }(t)=(\tau-t) / 2$ denotes the right endpoint of $J_{n}$. Differentiating (15) with respect to $t$, we obtain

$$
\partial_{t} z_{0}=\frac{\tau}{2\left(z_{0}+t\right)-e_{t}^{2} g^{\prime}\left(z_{0}+t\right)}-1
$$

Note that the derivative of $g$ must be positive. Thus using (9) we obtain that

$$
\tau-t-2 z+e_{t}^{2} g^{\prime}(z+t) \geq 0
$$

so that

$$
2(z+t)-e_{t}^{2} g^{\prime}(z+t) \leq \tau+t .
$$

On the other hand from the requirements on $C$ it follows that $C(u) \leq u$ and so

$$
g^{\prime}(u) \leq 2 C(u) C^{\prime}(u) \leq 2 C(u) \leq 2 u .
$$

Thus

$$
\partial_{t} z_{0}>\frac{\tau}{\tau+t}-1>-\frac{1}{2} .
$$


This implies that $\partial_{t} D$ is always positive. Hence $D(t)$ increases with $t$. Finally, note that $z_{0}(0)=0$ so $D(0)=-\tau / 2$, and that $D(\tau)=z_{0}(\tau)>0$. This means that, as $t$ increases from 0 to $\tau, z_{0}(t)$ will cross the left endpoint of the interval $J_{n}$ at least once (exactly once if $n$ is big enough) and its right endpoint exactly once. Thus a unique fixed point with jump number

$n$ appears at an inclination $t_{n}$ such that $z_{0}\left(t_{n}\right)=\sqrt{1-n^{-1}} z_{\max }\left(t_{n}\right)$ and ceases to exist for $t>t_{\infty}=\lim _{n \rightarrow \infty} t_{n}$.

We have thus established our first result: For every choice of $\tau, e_{t}$ and $g(u)$ [within the class considered here], there is an increasing sequence $\left\{t_{n}\right\}_{n=1}^{\infty}$ with a limit point

$$
t_{\infty}=\lim _{n \rightarrow \infty} t_{n}<\tau
$$

such that there is a fixed point with jump number $n$ for all $t \in\left(t_{n}, t_{\infty}\right]$. Furthermore the fixed point (for a given $n$ ) is unique.

\section{CONDITION FOR BOUNDED VELOCITY}

The dynamics of the map (14) can be analyzed using standard techniques in one-dimensional dynamical systems. For example, the orbit of a given point $z \in J_{n}$ can be obtained by setting $z_{1}=h(z), z_{2}=h\left(z_{1}\right)$, and so on. There is however a caveat about the dynamics of our map. Suppose that for a given point $z \in J_{n}$ its image $h(z)$ leaves the interval $J_{n}$. If we know $n$, then by (11) we see that $\mathcal{E}^{\prime}=n[h(z)]^{2}$. Thus, using (10), we determine $n^{\prime}$ by

$$
\left(n^{\prime}-1\right) z_{\max }^{2}<n[h(z)]^{2} \leq n^{\prime} z_{\max }^{2}
$$

or

$$
n^{\prime}=\operatorname{ceiling}\left[\frac{n[h(z)]^{2}}{z_{\max }^{2}}\right]
$$

Thus

$$
\begin{array}{ll}
n^{\prime}<n & \text { if } \quad[h(z)]^{2} \leq\left(1-\frac{1}{n}\right) z_{\max }^{2} \\
n^{\prime}>n & \text { if } \quad[h(z)]^{2}>z_{\max }^{2} .
\end{array}
$$

We are now in a position to discuss our next result: The particle velocity becomes unbounded whenever $t>t_{\infty}$ and stays (after a transient) uniformly bounded whenever $t<t_{\infty}$.

Suppose first that $t>t_{\infty}$ so that there is no fixed point in the interval $\left[0, z_{\max }(t)\right]$. By the assumptions on $C$, we have $h(z)>z$ for all $z$ in the 
physically relevant interval $\left[0, z_{\max }\right]$ and some iterate $h^{j}(z)$ is forced to leave the interval $J_{n}$ to the right. According to (25), the jump number and hence the particle velocity will increase indefinitely. Now suppose that $t<t_{\infty}$ so that $z_{0}(t)<z_{\max }(t)$. A typical graph of $h(z)$ in this case is shown in Fig. 2. Let $n_{0}$ then be the smallest integer satisfying: $z_{0}(t)<\sqrt{1-\left(n_{0}\right)^{-1}} z_{\max }(t)$, so that the intervals $J_{n}$, for $n \geq n_{0}$, will all lie to the right of $z_{0}$. This means that for $n \geq n_{0}$ we have $\mathcal{E}^{\prime}<\mathcal{E}$ and so $n^{\prime}<n$. For $n<n_{0}$, on the other hand, the jump number may grow (so that $n^{\prime}>n$ ). But from (23) we have that

$$
n^{\prime} \leq \max _{0<n<n_{0}} \sup _{z \in J_{n}(t)} \operatorname{ceiling}\left[\frac{n[h(z)]^{2}}{z_{\max }^{2}}\right] .
$$

Denoting the bound above by $n_{1}$, we then conclude that after a transient the jump number will be uniformly bounded by $\max \left\{n_{0}, n_{1}\right\}$.

The preceding argument thus proves the existence of the transition from bounded to unbounded velocity at $t=t_{\infty}$. In the region of bounded velocity, several dynamical regimes are possible depending on the stability of the fixed points, as we shall see next.

\section{STABILITY OF FIXED POINTS}

The fixed point $z_{0}(t)$ will be stable (unstable) whenever the derivative $\lambda(t)=h^{\prime}\left(z_{0}\right)$ satisfies the condition $|\lambda|<1(|\lambda|>1)$. The derivative at the fixed point is obtained from (12):

$$
\lambda(t)=\frac{e_{t}^{2} g^{\prime}\left(z_{0}+t\right)-2 t}{2 z_{0}} .
$$

From (19) it immediately follows that $\lambda<1$. Thus instability occurs if and only if $\lambda(t)<-1$. A straightforward calculation shows that $\lambda(0)=$ $\frac{1}{2} e_{t}^{2} g^{\prime \prime}(0)>0$, so the fixed point with $n=1$ is always born stable at $t=0$. As $t$ increases, this fixed point (and others as well) may remain stable or may eventually become unstable, depending on the nature of the restitution law.

From (27) we can see that the fixed points are always stable whenever the loss of tangential momentum (due to collisions) is small. In other words, if $e_{t}^{2} g^{\prime}(u)$ is not too far from $2 u$ we are guaranteed that $\lambda>-1$. When this is the case, then for $t_{2}<t<t_{\infty}$ we will have more than one attractor for the dynamics. On the other hand, for collision rules with large momentum loss (i.e., small $e_{t}$ ) there will be a critical inclination above which the fixed 
points become unstable. To see this, first consider the case $e_{t}=0$. Setting

$e_{t}=0$ in (15) yields: $z_{0}(t)=-t+\sqrt{t \tau}$. Substituting this into (27) gives $\lambda(t)=(1-\sqrt{\tau / t})^{-1}$. Thus for $t>\tau / 4$ we have $\lambda<-1$ and so the fixed are unstable. In particular, we note that for $e_{t}$ sufficiently small all fixed points with $n \geq 2$ are born unstable. This follows from the fact that for $e_{t}=0$ we have $t_{2}>\tau / 4$.

Let us now look at the transition to instability in more detail. In the $\left(e_{t}, t\right)$ plane we have found a region where $\lambda<-1$ (large $e_{t}$ ) and another one where $\lambda>-1$ (when $e_{t}$ is close to zero). These regions must be separated by the solutions of $\lambda\left(e_{t}, t\right)=-1$. In fact, setting $t=t\left(e_{t}\right)$ in (27), differentiating the relation $\lambda(t)=-1$ with respect to $e_{t}$, and using Eqs. (15) and (19), one can show that these solutions lie on a differentiable curve $t_{\text {inst }}\left(e_{t}\right)$. Thus for $t<t_{\text {inst }}$ the fixed point are stable whereas for $t>t_{\text {inst }}$ they become unstable.

We have thus seen that in the $\left(e_{t}, t\right)$ plane the regime of stable periodic orbits is bounded from above by a region of unbounded velocity (for large $e_{t}$ ) and by a region of unstable periodic orbits (for small $e_{t}$ ). These distinct regions can be most easily visualized in the context of a simple model [1] where the tangential restitution law is given by: $v_{x}^{\prime}=e_{t} v_{x}$, which means $g(u)=u^{2}$. In light of the preceding discussion, one can readily construct a phase diagram in the $\left(e_{t}, t\right)$ plane displaying all the possible dynamical regimes for this model [1]. This is shown in Fig. 3 for the case $\tau=1$. The four regions indicated in this figure correspond to:

I $-0<t<\min \left(t_{2}, t_{\text {inst }}\right)$ : there is a unique stable fixed point.

II $-t_{2}<t<\min \left(t_{\text {inst }}, t_{\infty}\right)$ : the system has multiple stable fixed points.

III $-t_{\text {inst }}<t<t_{\infty}$ : the fixed points are unstable.

IV $-t>t_{\infty}$ : no fixed point exists.

(When regions I and III overlap, there is a unique fixed point which is unstable.)

We have seen above that the nature of the phase diagram remains the same for all $g(u)$ within the general class discussed in this paper.

\section{CHAOTIC BEHAVIOR}

Let us now study the dynamics in the region where the fixed points are unstable. We are particularly interested in the limit $t \rightarrow t_{\infty}$ (from below). First we note that (since the velocity is bounded) for any orbit the jump number $n$ must (after possible transients) lie within a band: $n_{-}<n<n_{+}$. 
To obtain an estimate for this band we appeal to the inset in Fig. 2, where we show a blow-up of $h(z)$ near an unstable fixed point. In this inset we have indicated the points $z_{-}$and $z_{+}$, defined by $z_{-}=h\left(z_{\max }\right)$ and $h\left(z_{+}\right)=z_{\max }$. Now let $n_{-}$be the largest integer such that $z_{-}>\sqrt{1-\left(n_{-}\right)^{-1}} z_{\max }$, and $n_{+}$ the smallest integer such that $z_{+}<\sqrt{1-\left(n_{+}\right)^{-1}} z_{\max }$. By referring to Fig. 2 , one can convince oneself that if $n>n_{+}$so that $J_{n} \subset J_{n_{+}}$, then for any $z \in J_{n}$ its orbit will eventually leave the interval $J_{n}$ towards the left, thus decreasing $n$. Similarly, if $n<n_{-}$so that $J_{n_{-}} \subset J_{n}$, then for any $z \in J_{n}$ its orbit will eventually leave the interval $J_{n}$ towards the right and hence $n$ must increase.

From the inset of Fig. 2 one sees that the interval $\left[z_{+}, z_{\max }\right]$ is mapped to $\left[z_{-}, z_{\max }\right]$ and thus (asymptotically as $t \rightarrow t_{\infty}$ ):

$$
\left|\lambda\left(t_{\infty}\right)\right|=\frac{z_{\max }-z_{-}}{z_{\max }-z_{+}} .
$$

After performing a straightforward calculation one then obtains the following estimate (asymptotic as $t \rightarrow t_{\infty}$ ) for the band mean-value $\bar{n}=$ $\left(n_{+}-n_{-}\right) / 2$ and its width $\Delta n=n_{+}-n_{-}$:

$$
\begin{gathered}
\bar{n}=\frac{z_{\max }}{2\left(z_{\max }-z_{0}(t)\right)}, \\
\frac{\Delta n}{\bar{n}}=\frac{\left|\lambda\left(t_{\infty}\right)\right|-1}{\left|\lambda\left(t_{\infty}\right)\right|+1} .
\end{gathered}
$$

Within this band the dynamics is chaotic with a Lyapunov exponent approximately equal to $\ln \left|\lambda\left(t_{\infty}\right)\right|$. We note, however, that the orbits need not necessarily fill out the entire band. In fact, preliminary numerical results show that they fill out only a fraction of this band [5].

This work was supported in part by FINEP and CNPq.

\section{References}

[1] G. L. Vasconcelos and J. J. P. Veerman, Phys. Rev. E (in press).

[2] C. D. Jan, H. W. Shen, C. H. Ling, and C. I. Chen, in Proceedings of the 9th Conference of Engineering Mechanics, College Station, Texas, edited by L. D. Lutes and J. M. Niedzwecki (American Society of Civil Engineers, New York, 1992), pp. 768; G. H. Ristow, F.-X. Riguidel, and D. Bideau, J. Phys. I France 4, 1161 (1994). 
[3] S. F. Foerster, M. Y. Lounge, H. Chang, and K. Allia, Phys. Fluids 6, 1108 (1994).

[4] S. Dippel, G. G. Batrouni, and D. E. Wolf, Phys. Rev. E 54, 6845 (1996).

[5] C. G. Alves-Neto, G. L. Vasconcelos, and J. J. P. Veerman, unpublished. 


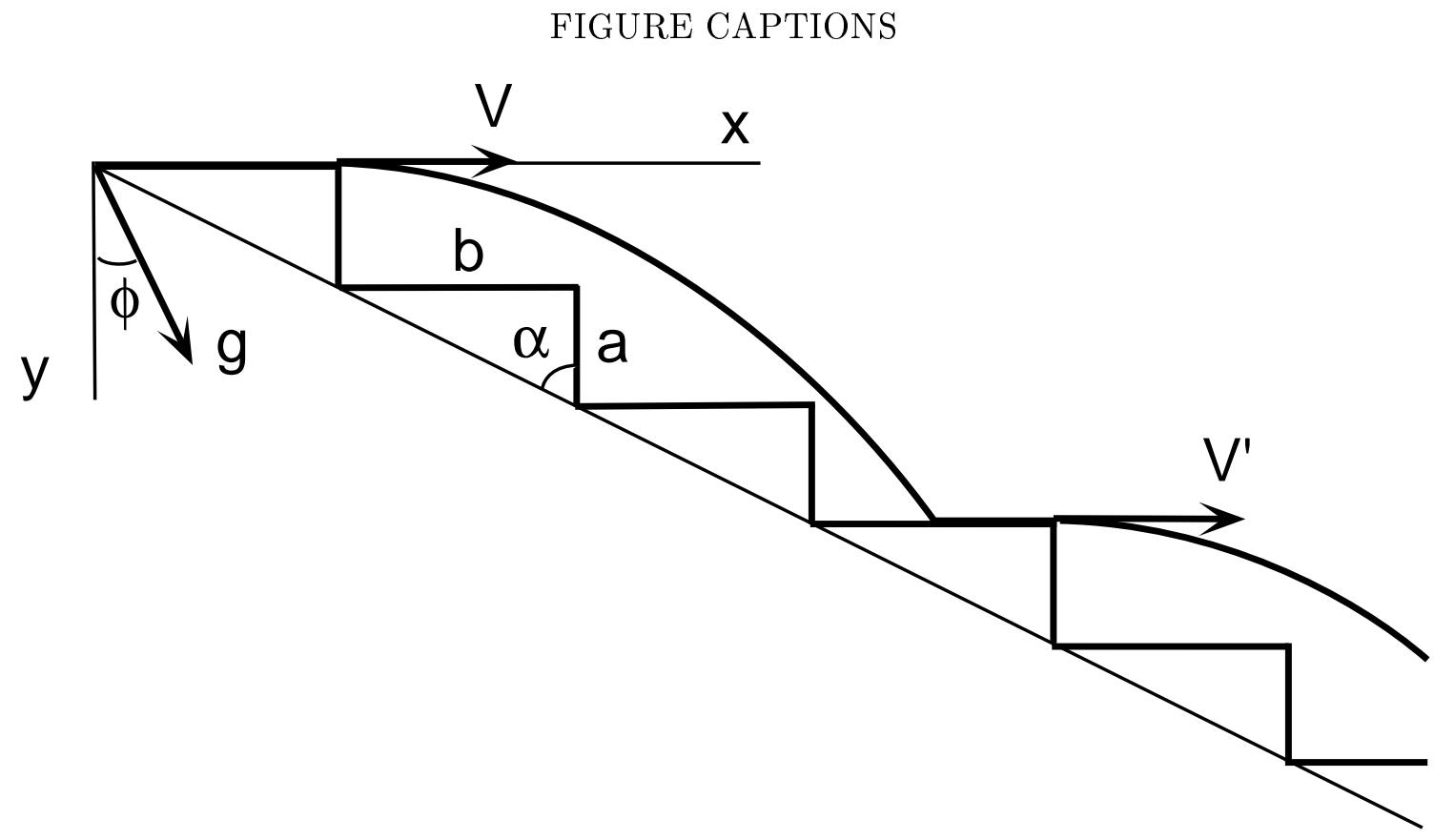

Figure 1: Model for a single grain moving on an inclined rough surface under gravity. 


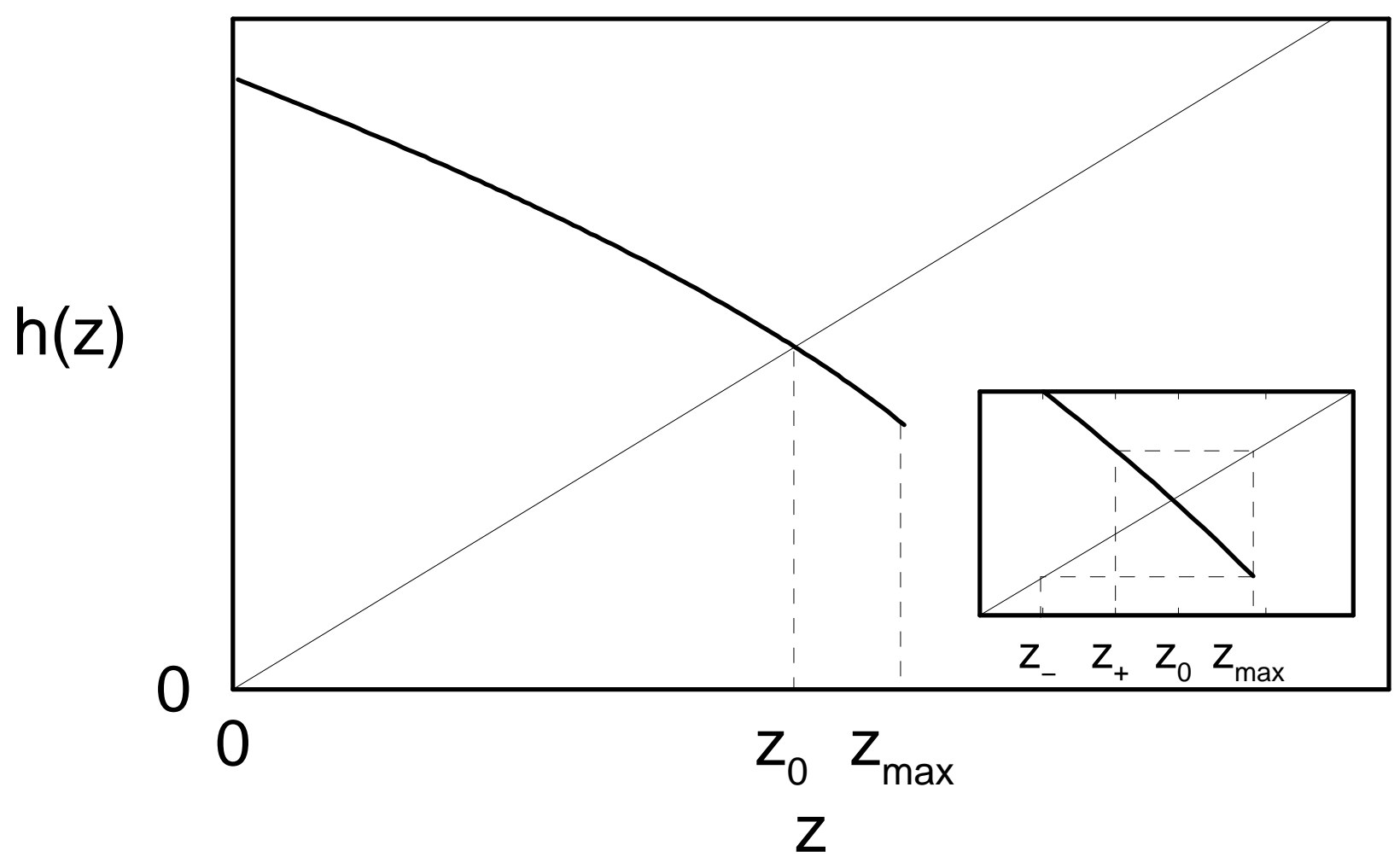

Figure 2: Graph of $h(z)$ showing the fixed point $z_{0}$. The inset shows a blow-up of a region near an unstable fixed point. 


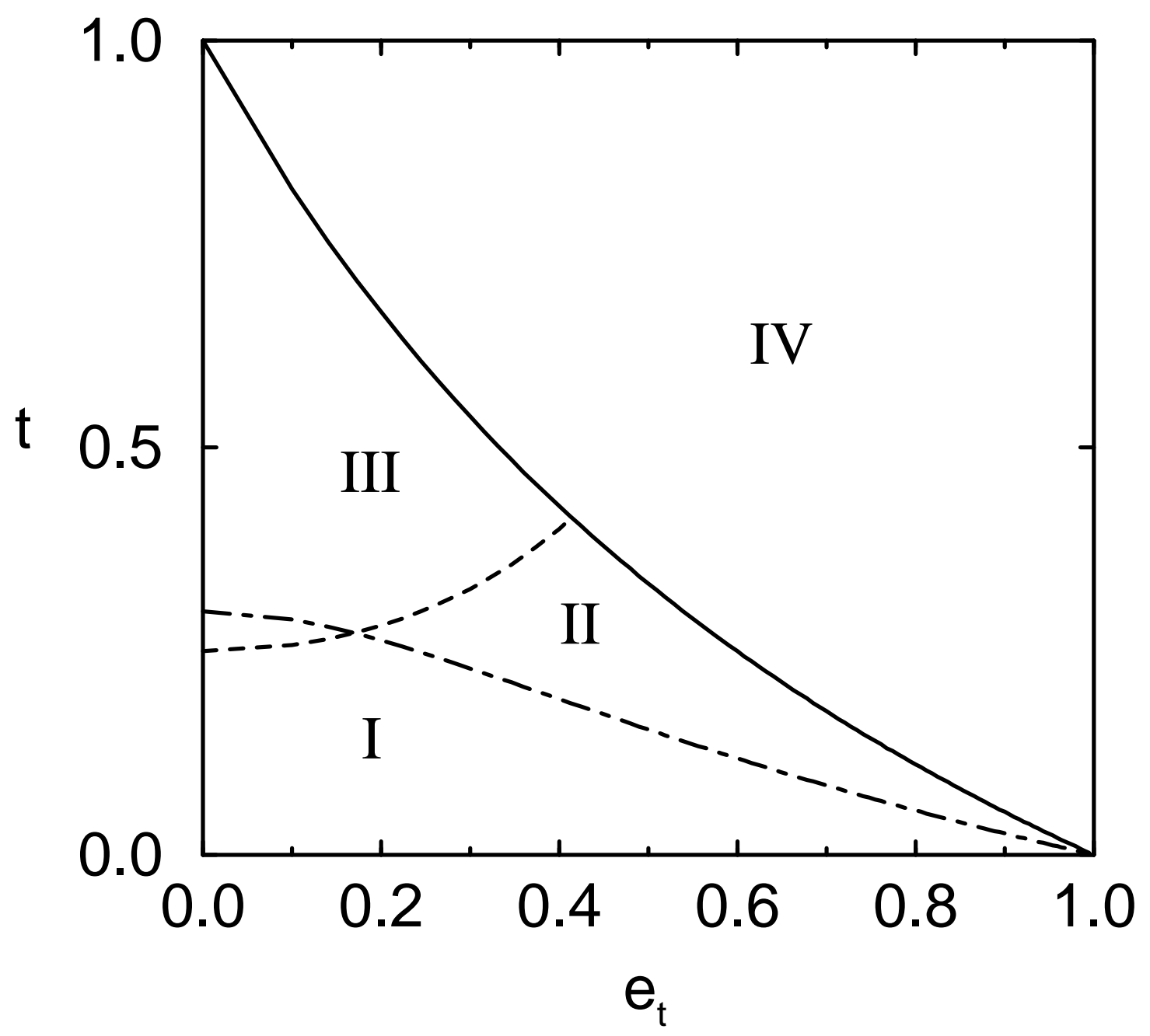

Figure 3: Phase diagram for the model with collision rule $v_{x}^{\prime}=e_{t} v_{x}$ and $\tau=1$. The solid line corresponds to $t_{\infty}$, the dashed line to $t_{\text {inst }}$, and the dot-dashed line to $t_{2}$. 